While we continue to work on how to improve the process even further, we feel that other hospitals can learn from what has already been achieved at Weston General Hospital. Given the improvements seen and that neutropenic sepsis mortality has fallen from 39 to $0 \%$, the culture at our hospital is that a neutropenic sepsis death should now be regarded as a 'never event'. Lessons learned have been fed back to other hospitals in our Strategic Clinical Cancer Network. ${ }^{11}$ -

\section{References}

1 National Institute for Health and Care Excellence. NICE Clinical Guideline 151. Neutropenic sepsis: prevention and management of neutropenic sepsis in cancer patients. London: NICE, 2012.

2 Kumar A, Roberts D, Wood KE et al. Duration of hypotension before initiation of effective antimicrobial therapy is the critical determinant of survival in human septic shock. Crit Care Med 2006;34:1589-96.

3 National Chemotherapy Advisory Group. Chemotherapy services in England: ensuring quality and safety. Sutton: NCAG, 2009.

4 National Confidential Enquiry into Patient Outcome and Death. For better, for worse? A review of the care of patients who died within 30 days of receiving systemic anti-cancer therapy. London: NCEPOD, 2008.

5 National Cancer Action Team. National Cancer Peer Review Programme 2004-2007. An overview of the findings from the second national round of peer reviews of cancer services in England. London: National Cancer Action Team, 2008.
6 National Cancer Action Team. National Cancer Peer Review Programme: acute oncology - including metastatic spinal cord compression measures. London: National Cancer Action Team, 2011.

7 National Cancer Action Team. Developing an acute oncology service: concepts and case studies. London: National Cancer Action Team, 2012.

8 UK Oncology Nursing Society. Acute oncology initial management guidelines. Marlow: UKONS, 2012.

9 Klastersky J, Paesmans M, Ruberstein EB et al. The multinational association for supportive care in cancer risk index: a multinational scoring system for identifying low-risk febrile neutropenic patients. J Clin Oncol 2000;18:3038-51.

10 Marshall E, Steven N, Billingham L et al. Difficulties in running a phase III trial to investigate the optimal management of patients with low-risk neutropenic sepsis. Poster presentation B137, NCRI Cancer Conference, 2-5 November 2014.

11 Wells T, Fountain V, Thomas C et al. Improving hospital mortality from neutropenic sepsis: lessons learned from a District General Hospital. South West Strategic Clinical Network Cancer Event, 8 July 2014

Address for correspondence: Dr TPE Wells, Weston General Hospital, Grange Road, Uphill, Weston-super-Mare BS23 4TQ, UK. Email: thomas.wells@nhs.net

\title{
The Royal College of Physicians' Fallsafe care bundles applied trustwide: the Northumbria experience 2013
}

\author{
Authors: David A Richardson, ${ }^{\mathrm{A}}$ Anju Bhagwat, ${ }^{\mathrm{B}}$ Kirsti Forster, ${ }^{\mathrm{C}}$ Ruth Hibbert, ${ }^{\mathrm{D}}$ Liam Robertson, ${ }^{\mathrm{E}}$ Philippa Whitelaw, ${ }^{\mathrm{F}}$ \\ Angela McArdle ${ }^{G}$ and Elizabeth Thompson ${ }^{H}$
}

The Royal College of Physicians' FallSafe care bundles constitute measures of good practice, some of which are recommended for all patients, some are additional measures for older and more vulnerable patients admitted to hospital, and there is another bundle for after an inpatient fall, to reduce the number of inpatient falls. In 2013 a dedicated

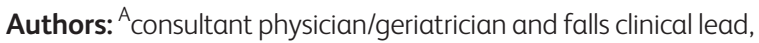

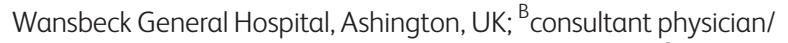
geriatrician, Wansbeck General Hospital, Ashington, UK; falls nurse practitioner, Wansbeck General Hospital, Ashington, UK; ${ }^{D}$ falls nurse practitioner, Wansbeck General Hospital, Ashington, UK; Efalls healthcare assistant, Wansbeck General Hospital, Ashington, UK; Ffalls nurse, Wansbeck General Hospital, Ashington, UK; ${ }^{G}$ falls administration support officer, Wansbeck General Hospital, Ashington, UK; ${ }^{H_{\text {falls }}}$ strategic lead, Wansbeck General Hospital, Ashington, UK healthcare assistant, trained by the falls team, started a monthly spot audit looking at preventative measures, on all inpatients on every ward of the trust. Monthly results were fed back to the ward managers, ward falls liaison nurses, doctors, therapists and pharmacy staff on each ward, to discuss at the monthly ward governance meetings. Training and advice on specific aspects of falls prevention were provided by falls nurse practitioners. In total, 9,679 patient episodes were recorded over the year. Compliance with the measures recommended by the FallSafe care bundles has improved following regular spot audit and training. This has led to an overall reduction in the number of inpatient falls. Despite this however, in the real world of changing patient demographics, ward closures and the increasing use of ambulatory care, the number of falls/1,000 bed days has increased.

KEYWORDS: Inpatient falls, FallSafe care bundles, Falls prevention 


\section{Introduction}

Over 280,000 patient falls are reported from acute, community and mental health units in England and Wales annually. ${ }^{1}$ They can cause serious injury and death and cost approximately $£ 15$ million per annum. ${ }^{2}$ Systematic review suggests multifactorial assessment and intervention can reduce falls by 20-30\%. ${ }^{3}$ The Royal College of Physicians' (RCP's) FallSafe quality improvement project took the successful components and formed them into care bundles to prevent and manage falls in clinical hospital wards in Southern England SHA., The FallSafe care bundles therefore represent measures of good practice, some of which are recommended for all patients, some are additional measures for older and more vulnerable patients admitted to hospital, and there is another bundle for after an inpatient fall, to reduce the number of inpatient falls (Box 1).

Northumbria Healthcare NHS Foundation Trust (NHFCT) includes three teaching district general hospitals and six

\section{Box 1. The FallSafe care bundles.}

\section{Bundle for all patients}

1. A history of previous falls and of fear of falling is taken at the time of admission.*

$>$ Admission processes and paperwork need to be changed to include these items.

2. Urinalysis is conducted on admission.

3. New prescriptions of night sedation are avoided.

4. A call bell is in reach.

> The existing call bell system must be able to reach all patient beds and chairs.

$>$ Systems are needed for rapid repair of faulty call bells.

5. Appropriate footwear is available and in use.

> Supplies need to be made available for patients without relatives or friends.

6. There is immediate assessment for and provision of walking aids.

> Physiotherapists must train nursing staff to provide appropriate walking aids at the time of admission to the ward, or as soon as they might be required.

$>$ Walking aids need to be made available for each ward area, and need a suitable storage area.

\section{Bundle for older and more vulnerable patients}

7. A cognitive assessment (mini-mental state examination (MMSE) or abbreviated mental test score (AMTS)) is conducted in all admissions aged $>70$ years.

8. Those at risk are tested for delirium (confusion assessment method).

$>$ Trusts must implement delirium screening as per NICE guidelines.

9. An assessment of risk versus benefit for use of a bedrail is conducted.

10. Visual assessment is conducted.

$>$ The ability to recognise objects from the end of the bed can be used as a screen for severe eyesight problems, and fuller assessment should be carried out if required.

11. Lying and standing blood pressure are taken with a manual sphygmomanometer.

12. Medication is reviewed with respect to cardiovascular and central nervous system acting medications.

> Nurses should request a review of medication to try and reduce the burden of drugs, particularly those associated with falls, and in patients who are unsteady, hypotensive or have orthostatic hypotension.

13. Based on observation, toileting arrangements are assessed and planned (tailored to needs rather than the standard two-hourly arrangement).

\section{Bundle for after a fall}

14. After a fall, appropriate assessments and procedures are followed, including neurological observations in those who have hit their head or had an unwitnessed fall.

> Trusts have been mandated to include these procedures within their policies by July 2011.

15. A post-fall review (how can further falls be prevented for this patient) is conducted.

16. A complete incident report (all falls) is created.

17. A root cause analysis (lessons to prevent falls for future patients) is carried out for severe harm falls.

*Long stay units may wish to amend to 'at least monthly' rather than 'on admission'; For rehabilitation units, community hospitals, stroke units, orthogeriatrics units, care of the elderly units and dementia units this should equate to all patients. In wards and units with a more mixed population, patients with a high vulnerability to falls are likely to be determined by local policy eg positive response to any of the NPSA 'four questions', total of Morse score or STRATIFY score, or all patients not fully independent and mobile. Reproduced with permission. ${ }^{8}$ 
community hospitals. There are 58 wards of varying age, design layout and specialty (including mental health), with a total of 1,048 beds (of which $34 \%$ are single rooms and only $13 \%$ of bed spaces are visible from a nursing station). We report the experience of the NHFCT falls team in trying to implement these recommendations into routine clinical practice across a large NHS foundation trust.

\section{Methodology}

The existing NHCFT mandatory falls training for nurses and healthcare assistants (HCAs), provided by members of the falls team and renewed three yearly, already focused on specific aspects of falls prevention (including documenting and acting upon the relevant sections of the Falls Care Plan and on the importance of accurate incident reporting). All information for the audit was therefore able to be retrieved from the existing medical, nursing and therapy documentation without the need for modification.

The FallSafe care bundles were modified and some measures adapted to ensure a trustwide ability to comply and to allow easier audit.

\section{Bundle for all patients}

1. A history of previous falls and of 'fear of falling' is taken at the time of admission: this audit measure was assessed by the information recorded in the nursing admission document or the accident and emergency (A\&E) admission document.

2. Urinalysis is conducted on admission: this measure was recorded from the A\&E admission document, medical assessment unit documentation or the nursing admission document.

3. No prescriptions of night sedation: in order for this to be performed relatively easily by the falls HCA, without access to a medication reconciliation databases, this measure relating to 'new prescriptions of night sedation' was adjusted to 'any prescription of night sedation'. This measure was collected from review of the drug kardex.

4. Appropriate footwear is in use: a significant number of elderly patients are admitted without suitable or appropriate footwear. For those without appropriate footwear, the trust purchased supplies of slipperettes. These come in two sizes and have a sole with rubber grips, which if worn appropriately, makes mobilising on the shiny hospital floors less hazardous. This measure was audited by assessing the patient at the patient's bed space.

5. A call bell is within site and reach: this measure was usually audited at the patient's bed space. Some patients, who were freely ambulant, were not included when using day rooms or sitting areas. However immobile patients, or patients who required assistance to mobilise, who were placed in such areas were audited.

6. Glasses are within site and reach: this measure was added into the care bundle for all patients and audited at the patient's bed space.

7. Mobility aids are within site and reach: this measure was also audited at the patient's bed space. We initially found there was a misplaced perception that by taking the walking aid away from a confused patient you will stop them attempting to mobilise. This had led to the walking aids being tidied at the end of the bay or deliberately placed away from the patient's immediate reach. Where this problem was encountered additional education was provided to the ward and therapy staff by the falls team.

\section{Bundle for older and more vulnerable patients}

8. Cognition assessment: this audit measure was assessed by reviewing the information recorded in the $\mathrm{A} \& \mathrm{E}$ admission document. This was part of an existing trust Commissioning for Quality and Innovation project (CQUIN).

9. Delirium assessment: this audit measure was assessed by reviewing the information recorded in the $\mathrm{A} \& \mathrm{E}$ admission document. This was part of an existing trust CQUIN.

10. Visual assessment: While this audit measure is in the care of the elderly nursing documentation, an actual visual assessment was rarely performed by nursing staff and instead the use of glasses was recorded. The physiotherapy teams were performing visual assessments, but were only initially documenting their findings for patients on the stroke pathway. By enlisting the physiotherapy team to document the visual assessments they perform on all patients, we were therefore able to assess this audit measure by reviewing the physiotherapy notes.

11. Bed rail assessment: assessed by reviewing the 'bed height, rail and mat matrix' included in the care of the elderly nursing documentation.

12. Lying and standing blood pressure: recorded using automated blood pressure monitoring devices as per a specific protocol included in the care of the elderly nursing documentation. Additional training and support was already in place for ward teams from the falls team prior to this audit.

13. Continence plan: assessed by reviewing the continence pathway included in the care of the elderly nursing documentation.

14. Cardiovascular or central nervous system (CNS) culprit medication: this measure was assessed by review of the drug kardex and comparing the medication list to the Medicines and falls in hospital: guidance sheet. ${ }^{6}$ This required additional training and support for the HCA from the falls team.

\section{Bundle for after a fall}

The falls team had previously modified the Nottingham University Hospitals' Medical report following a fall and produced a sticker, which is inserted into the medical notes and is completed by the doctor or nurse practitioner after reviewing the patient who has fallen. This sticker and the medical and nursing responses to the fall, were audited by the falls team nurse practitioners against the measures recommended.

\section{Outcome data}

Monthly audit results were fed back via e-mail to the ward managers, ward falls liaison nurses, doctors, therapists and pharmacy staff on each ward to discuss at the monthly ward governance meetings.

Data for falls and occupied bed days were collected from the trust incident reporting system and occupancy data, so that 'falls/1,000 bed days' could be calculated. When reporting an injury the National Patients Safety Agency classifications of harm were used. ${ }^{2}$ 


\section{Results}

\section{Bundle for all patients}

In total, 9,679 patient episodes were recorded over the year. Overall compliance with most measures improved (Fig 1). The percentage of all patients who received all 7 measures of inpatient falls prevention had risen from $6 \%$ to $38 \%$. Specifically, 5 of the 7 measures had shown an absolute percentage improvement in compliance (falls history 25\%; urinalysis $46 \%$; glasses within reach $2 \%$; walking aid within reach $14 \%$; no sedative medications $14 \%$ ), though 2 of the measures had no significant change from baseline (call bell within reach $-1.0 \%$; appropriate footwear $0 \%$ ).

\section{Bundle for older and more vulnerable patients}

3,276 patient episodes were recorded over 9 months (Fig 2). The percentage of patients who had received all 13 measures of inpatient falls prevention had risen from 0 to $11 \%$. All but one of the additional measures had shown improvement in compliance (dementia assessment 7\%; visual assessment 72\%; bedrail assessment 14\%; postural blood pressure recorded $20 \%$; continence assessment $32 \%$; cardiovascular/CNS culprit medication assessment $24 \%$; delirium assessment $-12 \%$ ).

\section{Bundle for after a fall}

687 inpatient falls were reviewed over the year (Fig 3). 9 of 11 post fall measures had improved (mobility, strength and balance review 5\%; impaired cognition review 15\%; delirium review $42 \%$; continence frequency/urgency review $19 \%$; risk vs benefit bedrails review 9\%; bone health/osteoporosis/fracture risk review 24\%; special observation consideration 33\%; impaired vision review $2 \%$; cardiovascular assessment $32 \%$ ) while 2 measures declined (medication review $-9 \%$; orthostatic blood pressure review -9\%). The percentage of all patients who had received all 11 post fall measures had risen from 5 to $17 \%$.

\section{Falls data}

The total number of inpatient falls fell by $6 \%$ in 2013 compared to $2012(3,386$ vs 3,607$)$. Similarly the number of patients who suffered moderate, serious or catastrophic harm as a result of an inpatient fall (death, fracture, subdural haematoma etc) fell by $4 \%$ (157 vs 150 ).

However the number of falls/1,000 bed days rose by $0.3 \%$ ( 9.1 to 9.4 ).

\section{Conclusions}

This is the first report on the effectiveness of the RCP FallSafe care bundles, when applied to all patients in a NHS foundation trust, to reduce inpatient falls. It demonstrates that the RCP FallSafe care bundles can be applied across a wide range of inpatient specialties, including acute geriatrics, geriatric rehabilitation and orthogeriatric wards, where older and more vulnerable patients are typically found and also which report the highest number of inpatient falls.

In order to implement the many measures in the RCP FallSafe care bundles the whole multidisciplinary team needed to be informed and involved, including the nursing staff, medical staff, therapists, pharmacists, estates and management. This also meant adaptation of some of the measures to make it practical to apply them across all wards and also easily auditable without any additional resources.

Some measures were already in place as part of a trust CQUIN (eg dementia, delirium assessment) or care of the elderly pathways (eg continence), and required no additional resources or time from the falls team, while others required additional education (eg walking aids) or were controversial and met with resistance from some medical teams (eg performing a routine urinalysis for fears of the indiscriminate use of antibiotics).

Some measures deserve specific comment. Continuous noninvasive beat-to-beat blood pressure monitoring is now the gold standard for accurately detecting postural hypotension

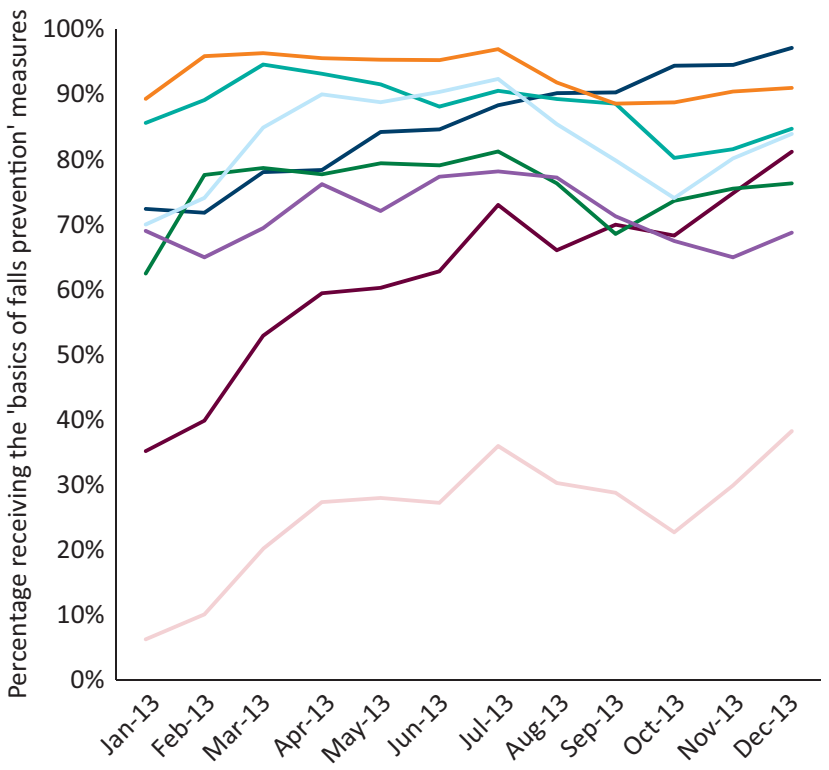

- Has been asked if any history of falls

— Urinalysis performed

- No hypnotic or sedative medication

- Appropriate fitting footwear or slipperettes

— Call bell within sight and reach

- Glasses within sight and reach

- Mobility aid within sight and reach

- Compliance with all 7 measures
Fig 1. Compliance with the 'Bundle for all patients' January-December 2013 $(n=9,679)$. 
Fig 2. Compliance with the 'Bundle for older and more vulnerable patients' AprilDecember 2013 ( $n=3,276)$.

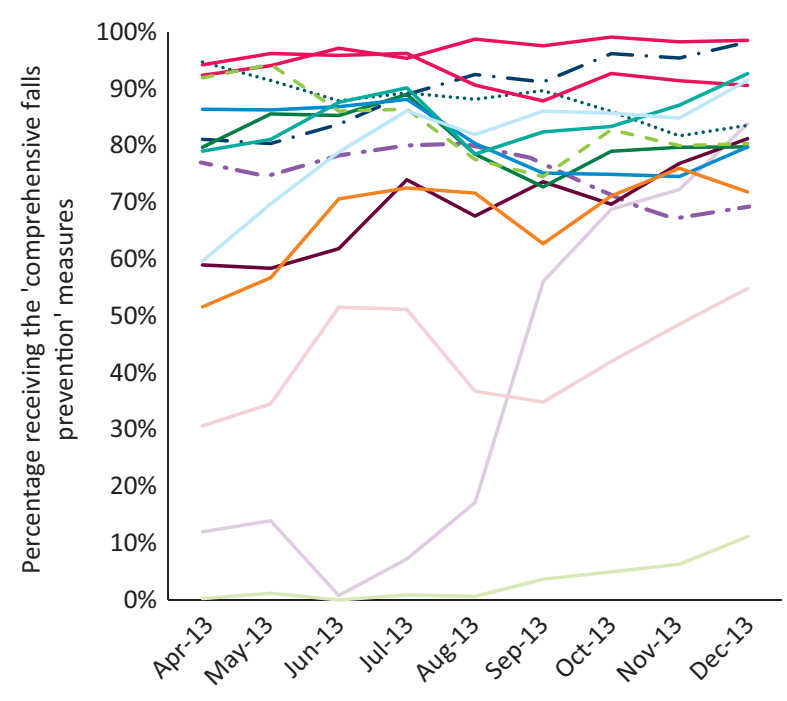

- Has been asked if any history of falls

— Urinalysis performed

- No hypnotic or sedative medication

- Appropriate fitting footwear or slipperettes

..... Call bell within sight and reach

_ Glasses within sight and reach

_ Mobility aid within sight and reach

_ Cognitive assessment

- - Delirium assessment

_ Visual assessment

_ Bed rail assessment

__ Lying and standing blood pressure Continence plan

Cardiovascular or CNS culprit medication

Compliance with all 14 measures in routine clinical practice. ${ }^{9}$ While this was available, it could not be provided everywhere in the trust. Using a manual sphygmomanometer requires specific training and is now no longer routinely performed on the wards due to the presence of automated blood pressure devices. We had therefore compromised and developed a protocol that could be applied on all wards, to all applicable patients and that would not be dependent on a few skilled staff that could use still use a manual sphygmomanometer. Therefore HCAs, after training and using standard automated blood pressure monitors, were asked to record three blood pressure measurements within the 3 minutes of standing to assess for evidence of postural hypotension. If there was a strong clinical suspicion for postural hypotension not detected by this method then patients could be referred to the falls team for consideration of non-invasive beatto-beat blood pressure monitoring.
It was recognised, that there are patient groups admitted to hospital where it is entirely appropriate to prescribe sedatives, or for them to be taking potentially 'culprit' cardiovascular or CNS medication. A ceiling effect was therefore reached early in the year, caused by the characteristics of the patients admitted, for these two measures. We did not assess whether medications were reviewed or adjusted; however work is ongoing with the pharmacy medication reconciliation teams to highlight these medications, so that the individual clinical teams can consider change if they feel appropriate.

There were also logistics problems with some measures. After an initial improvement in appropriate footwear, the supply of slipperettes ran out in the middle of the year and remained problematic for the rest of the year. This led to a fall in use of slipperettes and an increase in patients with no suitable or inappropriate footwear. A regular supply of
Fig 3. Compliance with the 'Bundle for after a fall' January-December 2013 $(n=687)$.

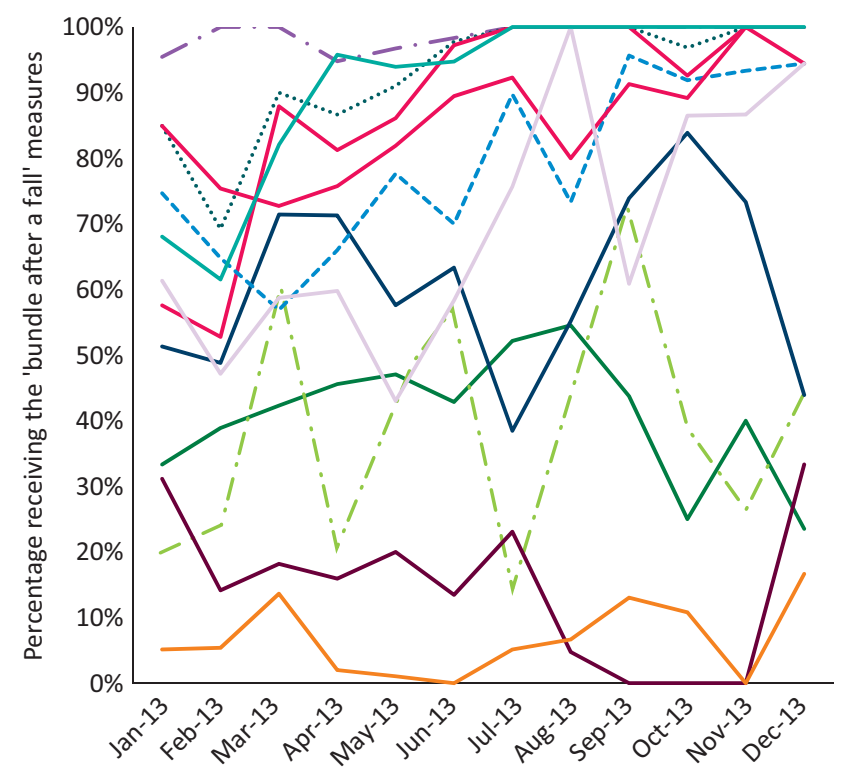

- Medication reviewed

_ Visual assessment

_ Orthostatic Hypotension assessment

- Mobility, Strength and Balance assessment

..... Cognitive assessment

— Delirium assessment

-.- Continence assessment

_ Bedrails assessment

- Bone Health assessment Special Observation assessment

_ Cardiovascular assessment

- All 
slipperettes has subsequently been funded by the trust and education regarding what constitutes suitable footwear communicated to patients, carers and staff through training and a publicity campaign.

Where required, providing patients with a suitable walking aid within 24 hours of admission to hospital, also was problematic. Ambulances tend to bring acutely ill patients into hospital without their usual walking aids, and therapy services, which can assess and provide suitable walking aids, are often limited at the weekend.

Although the trust has had a robust nurse-led patient incident reporting culture (reinforced during mandatory training and post-fall follow up) for many years, and we were confident all falls were reported, because we did not use the FallSafe under-reporting measure ${ }^{5}$ we cannot confirm whether this was actually the case. Therefore changes in reported falls may reflect more complete reporting. NHFCT has consistently reported higher than average inpatient falls/1,000 bed days data when compared nationally ${ }^{1,2}$ and we feel in some part this is due to the reporting culture. Falls/1,000 bed day rates are similar to that reported in wards equivalent to the FallSafe wards at the end of the intervention. ${ }^{5}$

The number of inpatient falls can be affected by many factors including environmental, staff and patient factors. A dedicated falls team is essential to provide the necessary training and support to implement the FallSafe care bundles. We modified our mandatory training to incorporate e-learning packages, work books and ward-based training to address the differing needs of the ward staff. A falls liaison nurse was appointed on each ward as ward 'falls champion' to help disseminate information and act as a learning resource for other ward members. During the study period 'intentional rounding' has been implemented by the trust, additional nursing staff rostered overnight and one-to-one nursing guidance implemented.

The trust has a high percentage of single rooms and nonvisible bed spaces. The falls team have looked at task lighting, flooring, bed rails, one-way glides, crash mats, and bed and chair alarms.

Pressures to reduce length of stay and hospital admissions has also led to a marked increase in the use of ambulatory care for the 'fitter' patients; therefore patients who are admitted to hospital tend to be frailer, have more comorbidity and be at a higher risk of an inpatient fall. We also had a number of ward closures, ward reconfigurations and long periods where patients were being 'boarded out' from their usual ward due to bed pressures. All these factors could contribute to the increased falls/1,000 bed days observed.

Compliance with the measures recommended by the FallSafe care bundles, has improved following regular spot audit and training. This has led to an overall reduction in the number of inpatient falls. Despite this, in the real world of changing patient demographics, ward closures and the increasing use of ambulatory care, the number of falls $/ 1,000$ bed days has increased. FallSafe advocates, falls team, trust board members and clinical commissioning groups should be aware of possible confounding influences and limitations when setting possible target measures for inpatient fall reduction.

\section{References}

1 National Patient Safety Agency. Slips trips and falls in hospital. London: NPSA, 2007.

2 National Patient Safety Agency. Slips trips and falls in hospital data update. London: NPSA, 2010.

3 Cameron ID, Gillespie LD, Robertson MC et al. Interventions for preventing falls in older people in care facilities and hospitals. Cochrane Database Syst Rev 2012;12:CD005465

4 The Health Foundation. Closing the gap through clinical communities; the FallSafe project. London: Health Foundation, 2012. Available online at www.health.org.uk/sites/default/files/CtGtCC_casestudies. pdf [Accessed 23 September 2015].

5 Healey F, Lowe D, Darowski A et al. Falls prevention in hospitals and mental health units: an extended evaluation of the FallSafe quality improvement project. Age Ageing 2014;43:484-91.

6 Royal College of Physicians. Medicines and falls in hospital: guidance sheet. London: RCP, 2012. Available online at www.rcplondon.ac.uk/ sites/default/files/documents/medicines-and-falls2.pdf [Accessed 23 September 2015].

7 National Patient Safety Agency. Essential care after an inpatient fall. Rapid response report. London: NPSA; 2011. Available online at www. rcplondon.ac.uk/sites/default/files/documents/rapid-response-reportessential-care-after-inpatient-fall.pdf [Accessed 23 September 2015].

8 Royal College of Physicians. The FallSafe care bundle. London: RCP, 2011.

9 Task Force for the Diagnosis and Management of Syncope; European Society of Cardiology; European Heart Rhythm Association; Heart Failure Association; Heart Rhythm Society; Moya A, Sutton R, Ammirati $\mathrm{F}$ et al. Guidelines for the diagnosis and management of syncope (version 2009). Eur Heart J 2009;30:2631-71.

Address for correspondence: Dr DA Richardson, Wansbeck General Hospital, Woodhorn Lane, Ashington NE63 9J], UK. Email: david.richardson@northumbria-healthcare.nhs.uk 